\title{
TESTES AMOSTRAIS PARA ESTUDO DA VEGETAÇÃO EM DUNAS DOMINADAS POR Ipomoea pes-caprae
}

\author{
PINHEIRO, G. F.; EMILIO, T. C. \& S.R. CUNHA \\ Laboratório de Ecologia da Vegetação Costeira. CTTMar, Universidade do Vale do \\ Itajaí, Itajaí, SC. Rua Uruguai, nº 458, 88302-202, Brasil. simone.cunha@univali.br
}

\section{RESUMO}

\begin{abstract}
Este trabalho teve como objetivo determinar a metodologia adequada para avaliação da abundância da vegetação em uma área monoespecífica de Ipomoea pes-caprae, utilizando como estudo de caso as dunas de Navegantes (SC, 2654'40"S e 48³9'00"W). A abundância foi determinada em termos de cobertura vegetal usando amostradores quadrados subdividido por fios de nylon. Um bastão foi colocado nas intersecções de fios e as espécies que tocavam o bastão foram registradas. Foram testados três tamanhos de amostradores: $1 \mathrm{~m}^{2}, 0,56 \mathrm{~m}^{2}$ e $0,25 \mathrm{~m}^{2}$. Para cada tamanho de amostrador, a cobertura foi analisada de duas maneiras: considerando todas as intersecções e, separadamente, em 30 intersecções selecionadas aleatoriamente. Foi determinada uma faixa de vegetação de $50 \mathrm{~m} \times 10 \mathrm{~m}$ na qual o amostrador foi posicionado 15 vezes, utilizando tabela de números aleatórios. Foram calculados média e erro padrão percentual para 2, 3, 4 até 15 repetições, para cada amostrador. Não houve diferenças nos valores de cobertura vegetal obtidos com a utilização de 30 ou com a utilização de todas as intersecções de fios dos amostradores. Isto ocorreu para os três tamanhos de amostrador testados. O percentual de cobertura apresentou resultados similares para os dois menores amostradores $\left(0,25 \mathrm{~m}^{2}\right.$ e $\left.0,56 \mathrm{~m}^{2}\right)$, com média de $30 \%$. Entretanto, o erro padrão do amostrador de $0,25 \mathrm{~m}^{2}$ foi em torno de $20 \%$, enquanto para os amostradores de $0,56 \mathrm{~m}^{2}$ e $1 \mathrm{~m}^{2}$, o erro foi de apenas $10 \%$. O valor de cobertura obtido com o amostrador de $1 \mathrm{~m}^{2}(40 \%)$ foi superior aos dois outros amostradores, devido à dificuldade de visualização das plantas nas intersecções, ocasionada pelo grande tamanho. Na estimativa do número necessário de réplicas para o cálculo da cobertura, a média e o erro percentual estabilizaram entre 6 e 8 réplicas para os amostradores de $0,56 \mathrm{~m}^{2}$ e $1 \mathrm{~m}^{2}$, enquanto que para o amostrador de $0,25 \mathrm{~m}^{2}$ foram necessárias 10 réplicas. Considerando estes resultados, concluímos que o melhor amostrador para avaliar o percentual de cobertura ou recobrimento é o amostrador de $0,56 \mathrm{~m}^{2}$, utilizando apenas 30 intersecções aleatoriamente escolhidas e 8 réplicas. Esta combinação possibilita uma avaliação adequada da vegetação, com o mínimo esforço possível, otimizando o processo de coleta de dados.
\end{abstract}

Palavras-chave: Dunas Costeiras, Amostragem, Ipomoea pes-caprae, Vegetação.

\section{SAMPLING DESIGN TO STUDY PLANT COMMUNITIES IN DUNES DOMINATED BY Ipomoea pes-caprae}

\begin{abstract}
This work aimed to determinate an adequate methodology to evaluate plant abundance in an area of Ipomoea pes-caprae, taking Navegantes dunes (SC, 2654'40"S e 48 $39^{\circ} 00^{\prime \prime} \mathrm{W}$ ) as a case study. Abundance as percentage of coverage was determinate using a sampler divided by nylon filaments. A stick was put at the filaments intersections and the species touching the stick was recorded. We tested 3 sampler sizes: $1 \mathrm{~m}^{2}, 0.56 \mathrm{~m}^{2}$ and $0.25 \mathrm{~m}^{2}$. For each sampler two different samplings were made: the species were recorded in all intersections and also in 30 randomly selected intersections. A dune zone $50 \mathrm{mX10m}$ was delimited, where 15 replicates for each treatment
\end{abstract}


were sampled. We calculated average of coverage and standard error as percentage, for 2 replicates, 3, 4, until 15 replicates, for each treatment. The use of all intersects or 30 intersections presented no influences on the results. Coverage percentages for the two smaller samplers were about $30 \%$, but standard error was higher to $0.25 \mathrm{~m}^{2}$ sampler (about $20 \%$ ), then to others (about $10 \%)$. Coverage percentage obtained by $1 \mathrm{~m}^{2}$ sampler was overestimated $(40 \%)$ due to difficulties to take a vertical look at the intersections far from the sampler edge. Six to eight replicates were needed when using the $0.56 \mathrm{~m}^{2}$ and $1 \mathrm{~m}^{2}$ samplers, and ten replicates were needed when using $0.25 \mathrm{~m}^{2}$ sampler. Considering these results, we concluded the best sampling is made using $0.56 \mathrm{~m}^{2}$ sampler, 30 intersections randomly selected and 6 to 8 replicates. These make possible to optimize the data collection with the minimum expenses of time and effort, and also the best description of the vegetation cover.

Key-words: Coastal Dunes, Sampling, Ipomoea pes-caprae, Dune Plants.

\section{INTRODUÇÃO}

Os ambientes de dunas são extremamente importantes, tanto sob o ponto de vista ecológico, como econômico. Devido a sua morfologia, as dunas agem como uma barreira natural, protegendo a linha da costa em eventos de tempestade ou ressacas, evitando inundações e erosões das áreas urbanizadas (Viles \& Spencer, 1995). Uma das maiores vantagens das dunas como protetora da linha da costa consiste na sua habilidade de recuperação natural. A forma mais fácil e rápida para a duna se reconstituir após uma ressaca ou por danos causado pelo homem é com o surgimento da vegetação (Hesp, 2001). A presença da vegetação resistente à alta salinidade favorece a retenção de sedimento, podendo reduzir fortemente o transporte de areia em direção ao continente (Hesp, 1991).

As dunas têm também grande importância como habitat natural de plantas, insetos, aves, répteis e outros organismos (Gianuca, 1997; Hesp, 2001). Além disso, a diversidade da flora e fauna das dunas e sua grande beleza paisagística representam um grande atrativo para o turismo, que é a industria que mais cresce no estado de Santa Catarina.

Com a ocupação de forma desordenada que o litoral catarinense vem sofrendo, tornase cada vez mais comum a construção civil sobre as dunas, e o uso destas áreas para lazer, muitas vezes sem planejamento ou de forma inadequada. Estas atividades reduzem a área de dunas e a vegetação, aumentando a exposição da costa às ações de fenômenos naturais e trazendo grandes prejuízos à população local, tanto devido à perda de proteção contra erosão e, em alguns casos até mesmo a perda de imóveis, quanto pela redução do potencial paisagístico e, conseqüente, pela redução das atividades turísticas.

Considerando a importância das comunidades vegetais para a manutenção das dunas costeiras, o conhecimento adequado desta vegetação é essencial para propor estratégias de conservação, recuperação e manejo destes ecossistemas. A utilização de técnicas não destrutivas é uma excelente ferramenta de avaliação e monitoramento da vegetação, especialmente em ambientes degradados e em estágio de recuperação, pois apresenta uma interferência mínima no ambiente. Entretanto, para uma representação confiável da comunidade vegetal, testes preliminares devem ser efetuados para avaliar o tamanho de amostrador, o número de pontos a serem contabilizados dentro do amostrador, além do número de réplicas necessárias para descrição da vegetação. Sendo assim, este trabalho tem como objetivo determinar uma metodologia adequada para avaliação da abundância da vegetação em áreas dominadas por de Ipomoea pes-caprae, utilizando como estudo de caso as dunas da porção sul da praia de Navegantes, SC. 


\section{ÁREA DE ESTUDO}

O estudo foi realizado na praia de Navegantes (SC, 2654'40 "S e 48॰39'00" W), que apresenta um estágio morfodinâmico dissipativo, possuindo cerca de $60 \mathrm{~m}$ de largura e 10.030 m de extensão (Menezes, 1999). Esta praia encontra-se orientada na direção Norte-Sul, estando exposta às ondulações provenientes dos quadrantes sul e sudeste, sendo caracterizada pela presença de um campo de dunas frontais bastante desenvolvido. $\mathrm{Na}$ porção mais ao sul, onde a praia limita-se com o molhe do Rio Itajaí-Açú, ocorrem dunas bastante modificadas morfologicamente. Nesta área a região frontal das dunas é colonizada por uma ampla faixa de Ipomoea pes-caprae.

\section{METODOLOGIA}

Neste estudo a abundância da vegetação foi estimada em termos de cobertura vegetal, utilizando-se amostradores quadrados, subdivididos com fios de nylon. Em cada intersecção de fios foi colocado um bastão, e foram registradas as espécies que tocavam o bastão. Os dados foram representados como percentual de recobrimento vegetal.

Foram testados: o tamanho do amostrador, o número de pontos (intersecções) dentro do amostrador considerado para estimativa da cobertura (todos os pontos ou 30 pontos selecionados aleatoriamente) e o número de réplicas que melhor representasse a comunidade vegetal. Foram testados três tamanhos de amostrador: $1 \mathrm{~m}^{2}, 0,56 \mathrm{~m}^{2}$ e $0,25 \mathrm{~m}^{2}$, subdivididos com fios de nylon. Os amostradores de $1 \mathrm{~m}^{2}$ e $0,56 \mathrm{~m}^{2}$ formaram 81 intersecções e o amostrador de 0,25m2 formou 64 intersecções. A cobertura vegetal foi efetuada considerando-se todas as intersecções de cada amostrador, e separadamente, considerando apenas 30 interseções selecionadas aleatoriamente e marcadas em cada amostrador. Cada um dos amostradores foi posicionado ale- atoriamente (conforme tabela de números aleatórios) 15 vezes em uma faixa de 50×10m (paralela $x$ perpendicular à praia) na região frontal das dunas, na qual ocorre uma comunidade monoespecífica de Ipomoea pes-caprae, e a amostragem foi efetuada.

Os dados referentes a cada tratamento $\left(0,25 \mathrm{~m}^{2}, 0,56 \mathrm{~m}^{2}\right.$ e $1 \mathrm{~m}^{2}$, com todas ou 30 intersecções) foram aleatorizados cinco vezes e, para cada uma, foram calculados os valores de média e de erro padrão percentual da cobertura vegetal, considerando-se 2 réplicas, 3 réplicas, 4, 5 e assim sucessivamente, até 15 réplicas.

Para a seleção do tamanho do amostrador, do número de intersecções e do número de réplicas, foram comparados os valores de média e de erro padrão percentual da cobertura vegetal, após a estabilização destes valores ao longo do número de repetições utilizadas.

\section{RESULTADOS}

Para os três tamanhos de amostradores testados, os resultados de cobertura vegetal obtidos com a utilização de todas as interseções foram similares aos obtidos com apenas 30 interseções.

O amostrador de $0,25 \mathrm{~m}^{2}$ apresentou para os dois tratamentos (todas e 30 intersecções) valores iguais de média e erro percentual para a cobertura vegetal (Figura 1), com média entre 20 e $35 \%$ e o erro percentual entre $20 \%$ e $25 \%$. Estes valores começaram a estabilizar a partir de 9 a 10 réplicas.

No amostrador de $1 \mathrm{~m}^{2}$, o percentual de cobertura médio foi de 35 a $50 \%$, com erro percentual entre 10 e $15 \%$ para os dois tratamentos (Figura 2), sendo estes estabilizados entre 8 e 10 réplicas. Com este amostrador, embora a estabilização da média e do erro padrão percentual possibilite o uso de um menor número de réplicas em comparação ao amostrador de $0,25 \mathrm{~m}^{2}$, o percentual de cober- 
tura foi superestimado, devido à dificuldade de visualização das plantas nas intersecções, ocasionada pelo seu grande tamanho e portanto, a impossibilidade de um posicionamento perpendicular a cada intersecção para contagem das plantas.

No amostrador de $0,56 \mathrm{~m}^{2}$, a cobertura percentual média foi de 25 a $30 \%$ para os dois tratamentos (todas as intersecções e 30 selecionadas aleatoriamente) e o erro percentual foi de 10 a $15 \%$ (Figura 3). A estabilização de valores foi observada entre 6 e 8 réplicas. Com este amostrador houve uma rápida convergência de valores, especialmente da média de cobertura vegetal, que apresentou valores intermediários entre os dois outros amostradores. Além disto, foi possível manter um baixo erro padrão com um número menor de réplicas, quando comparado aos dois outros amostradores.

\section{DISCUSSÃO}

Conseguir amostrar adequadamente a variabilidade natural do ambiente de estudo com o mínimo esforço amostral é sempre um grande desafio nos estudos ecológicos, uma vez que a obtenção de dados geralmente consome grande quantidade de tempo e envolve atividades de campo e/ou laboratório bastante laboriosas e extenuantes. As amostragens piloto e os testes amostrais, embora também laboriosos, são essenciais para a otimização da aquisição de dados, possibilitando a coleta
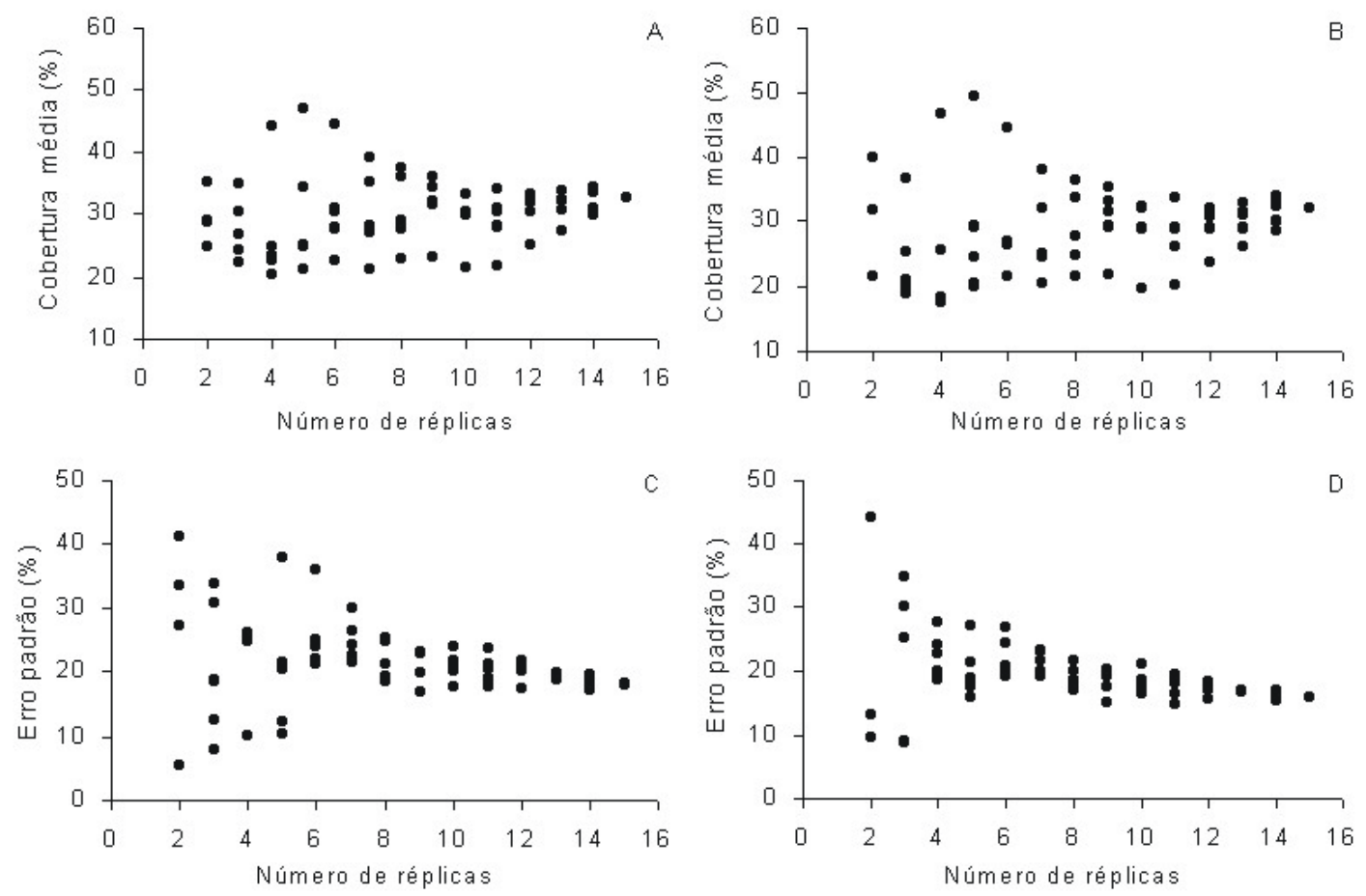

Figura 1 - Amostrador de 0,25m²: A) Cobertura vegetal média considerando todas as intersecções do amostrador; B) Cobertura vegetal média considerando 30 intersecções; C) Erro padrão percentual considerando todas as intersecções; D) Erro padrão considerando 30 intersecções. 
de maior quantidade de dados de qualidade, com o mínimo gasto de tempo e esforço.

A influência do tamanho do amostrador é bem reconhecida em estimativas de biomassa e de densidade de indivíduos (Barbour et al., 1987; Ludwig \& Reynolds, 1988; Krebs, 1989; Jogman et al., 1995). Entretanto, quando o parâmetro a ser estimado é a cobertura vegetal (ou percentual de recobrimento) a área do amostrador geralmente tem pouca influencia sobre os valores observados (Barbour et al., 1987).

Neste trabalho os valores de cobertura vegetal (média e erro padrão) foram influenciados pelo tamanho do amostrador, a despeito do que é geralmente assumido na literatura. Considerando que a metodologia exige a observação vertical da intersecção de pontos para contabilizar as espécies que tocam o bastão, o amostrador de $1 \mathrm{~m}^{2}$ produziu valores superestimados de cobertura devido às dificuldades em se observar perpendicularmente os pontos mais distantes da borda do quadrado. Este amostrador também apresenta maior dificuldade de manuseio e transporte no campo, o que resulta em maior gasto de tempo para a amostragem. $\mathrm{O}$ amostrador de $0,25 \mathrm{~m}^{2}$ também não foi muito eficiente na determinação da cobertura vegetal, apresentando um erro percentual elevado, e necessitando de maior número de réplicas, em comparação aos outros dois amostradores. Certamente isto é conseqüência da proporção de tamanho do amostrador em relação ao porte das plantas, $\mathrm{e}$ também é conseqüência do padrão de distribuição da vegetação apresentar uma tendên-
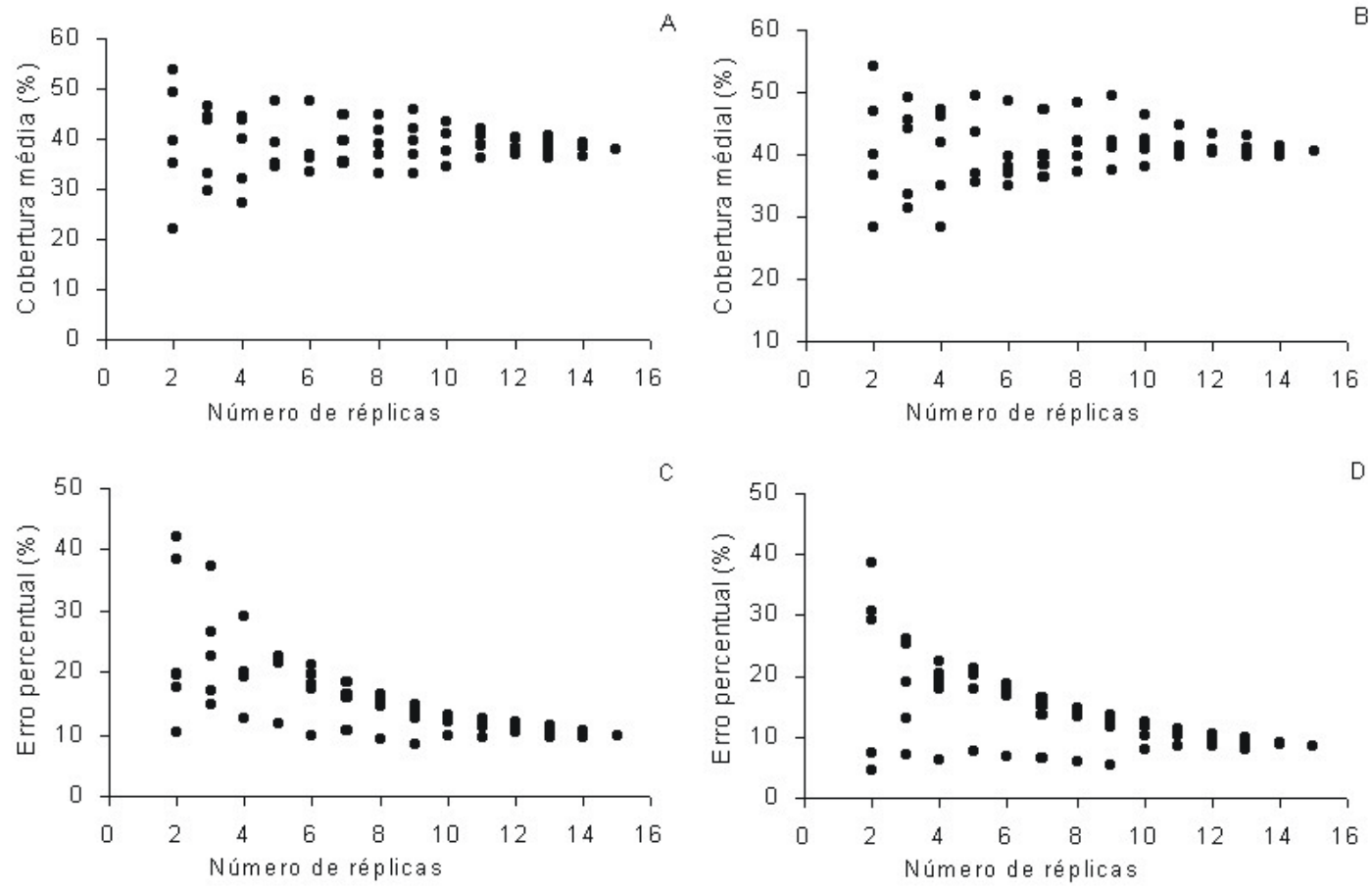

Figura 2 - Amostrador de $1 \mathrm{~m}^{2}$ : A) Cobertura vegetal média considerando todas as intersecções do amostrador; B) Cobertura vegetal média considerando 30 intersecções; C) Erro padrão percentual considerando todas as intersecções; D) Erro padrão considerando 30 intersecções. 
cia à distribuição agrupada, em função de seu modo de crescimento.

Embora neste tipo de amostragem (percentual de recobrimento ou cobertura) o tamanho do amostrador não apresente influência direta no esforço amostral (tempo de amostragem por réplica), ficou bastante clara a necessidade de testes preliminares, uma vez que o amostrador de tamanho intermediário possibilitou a obtenção dos melhores resultados em termos percentual médio de cobertura, de erro percentual e de número de réplicas. $O$ número de intersecções a serem observadas em cada amostrador, por outro lado, representa um elevado consumo de tempo. Entretanto, como os testes demonstraram que os resultados de cobertura vegetal (média, erro padrão percentual e número de réplicas necessárias) a partir da contagem de espécies em 30 intersecções selecionadas aleatoriamente foram similares aos resultados obtidos pela contagem de todas as intersecções (64 no menor amostrador e 81 nos outros dois), o uso de um menor número de intersecções representa uma importante economia de tempo durante as amostragens.

\section{CONCLUSÕES}

Considerando os resultados obtidos neste trabalho, concluímos que a amostragem não destrutiva da cobertura vegetal em áreas monoespecíficas das dunas pode ser otimizada com a utilização de um amostrador de $0,56 \mathrm{~m}^{2}$, registrando-se as espécies que tocam o bas-
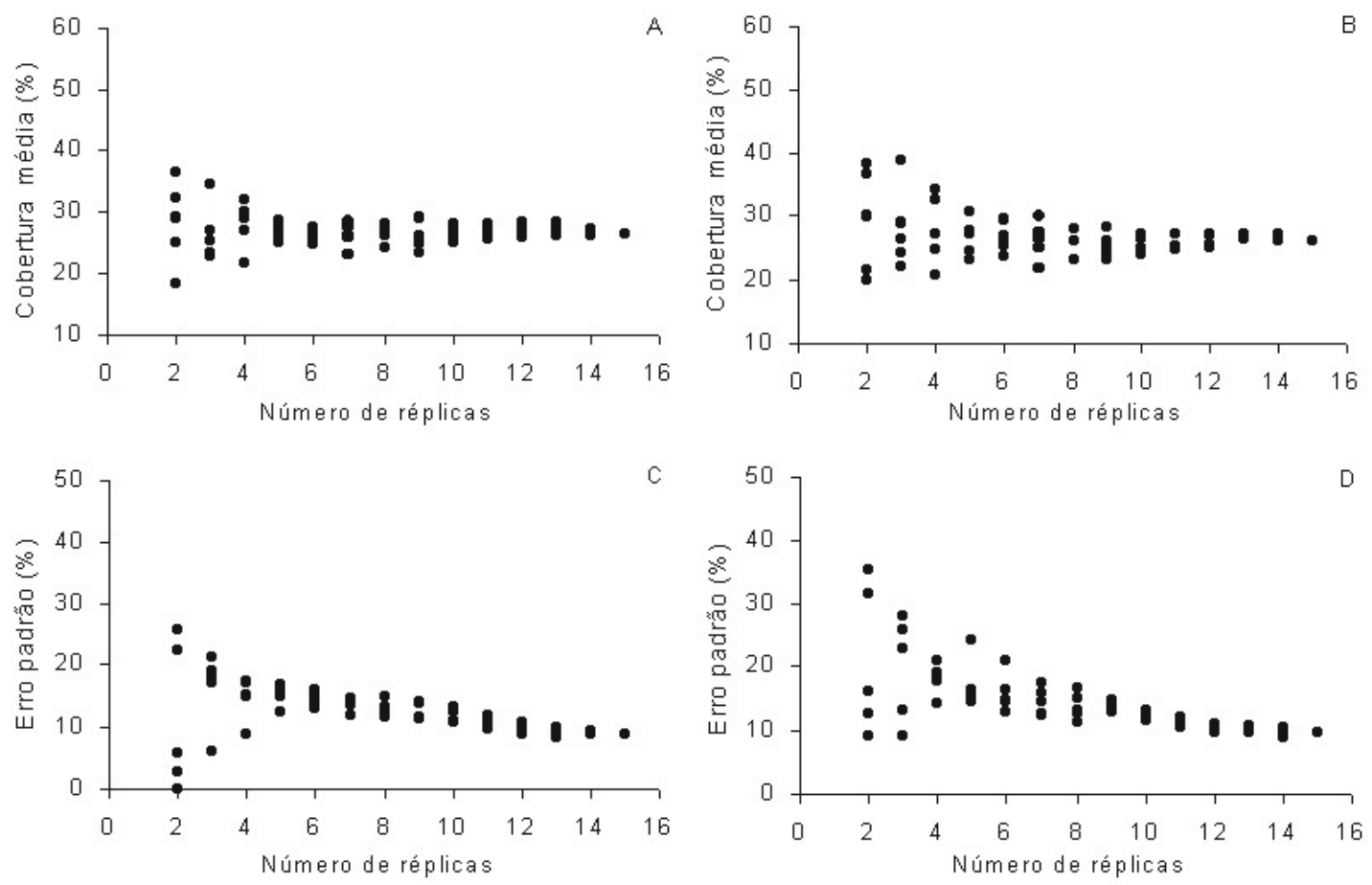

Figura 3 - Amostrador de 0,56 $\mathrm{m}^{2}$ : A) Cobertura vegetal média considerando todas as intersecções do amostrador; B) Cobertura vegetal média considerando 30 intersecções; C) Erro padrão percentual considerando todas as intersecções; D) Erro padrão considerando 30 intersecções. 
tão em 30 intersecções selecionadas aleatoriamente. Concluímos também que oito réplicas são suficientes para representar adequadamente a cobertura vegetal, com um erro percentual abaixo de $15 \%$.

\section{REFERÊNCIAS BIBLIOGRAFICAS}

Barbour, M. G.; J. H. Burk \& W. D. Pitts. 1987. Terrestrial plant ecology. 2nd Ed. Canada: The Benjamim/Cummings Publishing Company.

Gianuca, N. M. A. 1997. Fauna das Dunas Costeiras p. 114-116. In: Seeliger, U.; Odebrecht, C. e Castello, J.P. (eds.) Os Ecossistemas Costeiro e Marinho do Extremo Sul do Brasil. Editora Ecoscientia, Rio Grande.

Hesp, P. A. 1991. Ecological processes and plant adaptations on coastal dunes. Jornal of Arid Environments, Australia, 165-191.
Hesp, P. A. 2001. Coastal Sand Dunes: Form and function. Coastal Dunes Vegetation Network Technical bulletin. 4th ed. Nova Zelândia: Coastal Dunes Vegetation Network, 28p.

Jongman R. H. G.; C. J. F. Ter Braak. \& O. F. R. Van Tongeren. 1995. Data analysis in community and landscape ecology, Ed. Cambridge University Press.

Krebs, C. J. 1989. Ecological Methodology. New York:Harper \& Row Publisher.

Ludwig, J. A. \& J. F. Reynolds. 1988. Statistical ecology. A primer on methods and computing, Ed. John Wiley \& Sons.

Menezes, J. T. 1999. Aspectos Morfodinâmicos das Praias do Litoral centro-norte catarinense. Tese de graduação, Itajaí, Universidade do Vale do Itajaí.

Viles,H. \& T. Spencer. 1995. Coastal problems. Geomorphology, Ecology and Society at the coast. Edward Arnold. 